\begin{tabular}{|c|c|c|}
\hline Beitr. Ent. & Keltern & ISSN 0005-805X \\
\hline $\mathbf{5 2}(2002) 2$ & S. $407-415$ & 16.12 .2002 \\
\hline
\end{tabular}

\title{
Morphologie und Histologie der Speicheldrüsen der männlichen Skorpionsfliege Panorpa vulgaris IMHOFF \& LABRAM
}

\section{(Insecta: Mecoptera, Panorpidae)}

Mit 6 Figuren und 1 Tabelle

\author{
ALI AGHA NAHIF
}

\section{Zusammenfassung}

Die paarige männliche Speicheldrüse von Panorpa vulgaris (Mecoptera, Panorpidae) wird morphologisch und histologisch beschrieben. Diese Drüse besteht aus je 2-4 blind endenden Drüsenschläuchen. Sie sezernieren das Sekret-Bonbon als Hochzeitgeschenk. Die Entwicklung und der Füllungsgrad der Drüsen und der Aufbau des Drüsenepithel der Imagines hängt von der aufgenommenen Nahrung während der Larvalphase ab. Die Befunde zeigen, dass die Drüsenschläuche adulter Panorpen sowohl bei ungefütterten als auch bei gefütterten Exemplaren gleich stark entwickelt sind. Mit histochemischen Färbungen konnte nachgewiesen werden, dass diese Drüsenzellen ein proteinhaltiges Sekret enthalten.

\section{Summary}

The morphology and histology of the salivary glands of the males of Panorpa vulgaris (Mecoptera, Panorpidae) are described. Each of the two male salivary glands consists of 2-4 appendix tubes and produces a secrete intended as a "courtship meal". The development and the filling of the glands, as wellas the structure of the gland epithel of adult scorpion flies depend on the food intake during the larval phase, since the glands of adult Panorpa developed equally well under both feeding regimes. The glands produce a secrete which reacts positively to histochemical protein staining.

\section{Key words}

Mecoptera, Panorpidae, scorpion flies, Panorpa vulgaris, morphology, histology of salivary glands, nuptial feeding

\section{Einleitung}

Die Mecoptera (Schnabelfliegen) umfassen insgesamt neun Familien mit 32 Gattungen und etwa 500 Arten (BYERS \& THORNHILL 1983).

KALTENBACH (1978) beschreibt in seiner umfassenden Monographie über die Mecoptera die Morphologie, Histologie, Physiologie und Ökologie der Panorpidae; zur Morphologie der Speicheldrüsen werden kurze Ausführungen gemacht. 
Von den weltweit 150 Arten der Panorpidae kommen im Mitteleuropa lediglich 5 Arten der Gattung Panorpa vor. Die Skorpionsfliegen spielen in der gemäßigten Klimazone die ökologische Rolle der "Hygiene-Polizei". Sie ernähren sich vorwiegend von Insektenaas und fressen nur gelegentlich geschwächte oder weichhäutige Insekten. Außerdem nehmen sie auch Früchte, Nektar und Honigtau zu sich. Skorpionsfliegen findet man vor allem an schattigen, feuchten und mit Pflanzen besiedelten Stellen.

Die Morphologie und Entwicklung von $P$. vulgaris ist gut bekannt (NAHIF \& MADEL 1995). Die Entwicklung läuft über 4 Larvenstadien. Die Larven sind oligopod, langgestreckt und raupenähnlich. Die Puppe ist eine Pupa dectica.

Das Männchen bietet dem Weibchen zur Kopulation ein eiweißhaltiges, tropfenförmiges Sekret-Bonbon an, dessen Substanzen in den Speicheldrüsen gebildet werden. Die Fähigkeit eines Männchens, derartige Sekret-Bonbons zu produzieren, steht in direktem Zusammenhang mit der Nahrungsverfügbarkeit (FLECK u. a., 1994). Die Männchen von P. vulgaris bieten fertilen Weibchen eine größere Anzahl von Sekret-Bonbons an (SINDERN u. a., 1995).

In der vorliegenden Arbeit werden Untersuchungen über die Speicheldrüsen der männlichen Skorpionsfliege $P$. vulgaris vorgestellt. Obwohl die Speicheldrüsen von einigen Arten der Panorpiden, darunter auch Panorpa communis L., untersucht wurden (GRELL 1936), liegen über Morphologie und Histologie der Speicheldrüsen der Skorpionsfliege P. vulgaris bisher keine Angaben in der Literatur vor.

\section{Danksagung}

Ich danke den Herm Dr. GÜNTER MADEL und Privatdozent Dr. KLAUS REINHOLD (Institut für Evolutionsbiologie und Zooökologie der Universität Bonn) für die kritische Durchsicht des Manuskriptes.

\section{Material und Methoden}

Die Tiere stammen aus der Zucht, die im Institut für Evolutionsbiologie und Zooökologie in Bonn seit 1995 etabliert ist. Die Aufzucht der Larven und die Haltung der Imagines von $P$. vulgaris wurde von SAUER \& HENSLE (1977) beschrieben.

Für die hier beschriebenen Untersuchungen der männlichen Speicheldrüsen von $P$. vulgaris erfolgte die Haltung der frisch geschlüpften Imagines separat in gekennzeichneten Drosophila-Gläsern ( $5 \mathrm{~cm}$ Durchmesser, $10 \mathrm{~cm}$ Höhe) im Labor. Um den Tieren genügend Feuchtigkeit zu bieten, wurde der Boden der Gefäße mit naßfeuchten KleenexTüchern belegt.

Die männlichen Skorpionsfliegen wurden wie folgt ernährt: Die 1. Gruppe ohne, die 2. Gruppe mit einem und die 3. Gruppe mit zwei mittleren Abdominalsegmenten der Larve des Mehlkäfers Tenebrio molitor.

Die Tiere wurden 3, 6, 9, 12, 15 bzw. 18 Tage nach der Adulthäutung abgetötet und aufpräpariert. Die Präparation der Tiere erfolgte in einer kalten 0,65\%igen NaCl-Lösung. Die isolierten Speicheldrüsen wurde in Carnoy, Bouin bzw. in Formol nach BAKER (ROMEIS 1989) bei $+4{ }^{\circ} \mathrm{C}$ fixiert. Totalpräparate von Speicheldrüsen wurden mit Boraxcarmin angefärbt (ROMEIS 1989). Von den in Paraffin eingebetteten Speicheldrüsen wurden $5 \mu \mathrm{m}$ dicke Schnitte hergestellt und mit Giemsa, Hämalaun-Eosin, 
Eisenhämatoxylin bzw. Azan nach Heidenhain und einer modifizierten Azanfärbung nach SPECHT (ROMEIS 1989) gefärbt. Zur Färbung der $2 \mu \mathrm{m}$ dicken Semidünnschnitte (PLATTNER 1981) wurde 1-2\%iges Toluidinblau (ROMEIS 1989) verwendet.

Die Speicheldrüsensekret von P. vulgaris wurde histochemisch auf Kohlenhydrate, saure Mucopolysaccharide, Proteine und Lipide untersucht (RoMEIS 1989, RUTHMANN 1966, SPANNHOFF, 1967). Die gesamten Färbungen zur Darstellung von Kohlenhydraten und Proteinen wurden an den $5 \mu \mathrm{m}$ dicken Paraffinschnitten durchgeführt. Zum Nachweis der Lipide wurde reines Sekret verwendet.

Die fotografische Dokumentation der morphologischen und histologischen Präparate erfolgte mittels eines Universalmikroskops (Leitz).

\section{Ergebnisse}

Bei der Präparation fallen die Speicheldrüsen (= Labialdrüsen) durch ihre glasige Beschaffenheit und die gelblich-weiße bis bläuliche Farbe auf. Sie bestehen aus Drüsenschläuchen, Reservoir, Ausführgang und Speichelpumpe (Fig. 1). Mit Boraxcarmin färben sich die Drüsenschläuche kräftig rot an (Fig. 2A-B).

Die paarig entwickelte Speicheldrüsen bestehen aus 2-8 blind endenden z.T. divertikelbesetzten, Drüsenschläuchen (beidseitig 2-4) (s. Tab. 1), die unterhalb des Ösophagus liegen (Fig. 3A). Sie sind ca. 7,5 mm lang und reichen damit bis zum letzten Abdominalsegment. Sie nehmen im Körper des Männchen, neben dem Darmtrakt, von allen Oganen den meisten Platz in Anspruch. Sie sind von Fettkörpern und Tracheen umgeben. An ihnen inserieren weder Nerven noch Muskeln.

Auch bei der Speicheldrüsen von $P$. communis fehlen Muskelansätze. Stattdessen besitzen sie einen Verschlußmechanismus, der die Funktion eines Ventils übernimmt (GRELL 1936).

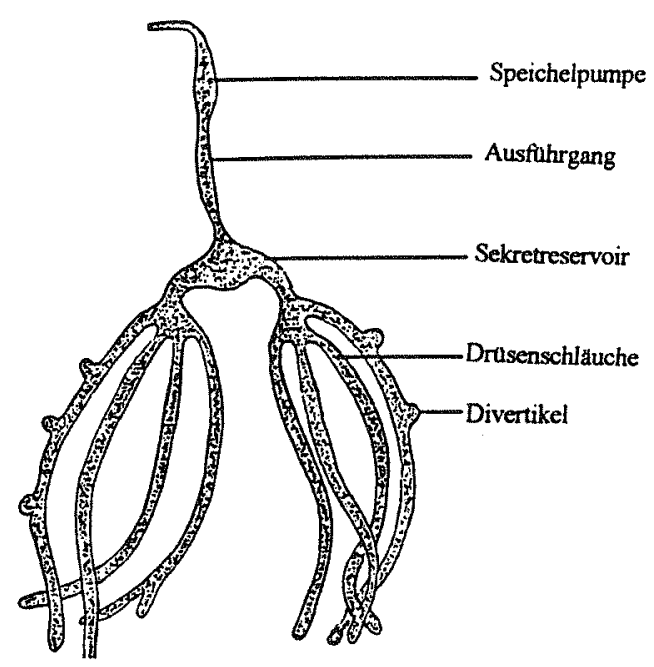

Fig. 1: Panorpa vulgaris Schematische Darstellung der männlichen Speicheldrüsen.
Die einzelnen Drüsenschläuche von $P$. vulgaris vereinigen sich und münden kurz danach im Bereich des Schlundganglions in ein gemeinsames Reservoir. An das Reservoir schließt sich ein langer Ausführgang an, der vor seiner Mündung eine Speichelpumpe besitzt. Das Sekret wird durch Haemolymphdruck mittels der Speichelpumpe zur Mündung herausgepreßt.

Wie histologisch nachweisbar, haben die Speicheldrüsen von $P$. vulgaris ein einschichtiges, hochprismatisches Epithel, das sich kurz vor der Mündung in das gemeinsame Reservoir verjüngt. Das Cytoplasma der Zelle ist granuliert und enthält einen $11 \mu \mathrm{m}$ großen, runden bis ovalen, exentrisch gelagerten Kern, der große Chromatinpartikel enthält. Die 


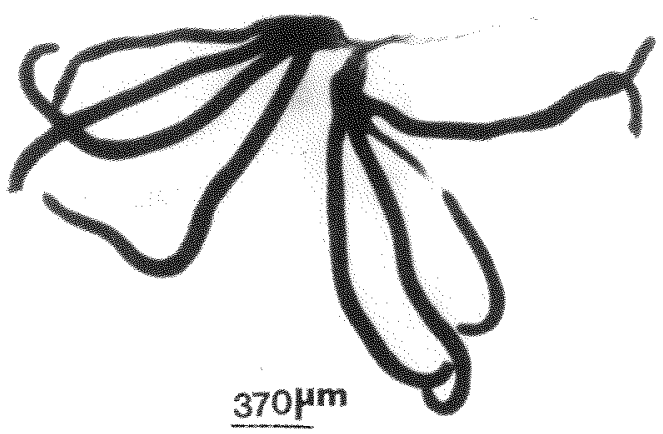

A

$\underline{370 \mu m}^{\mu m}$
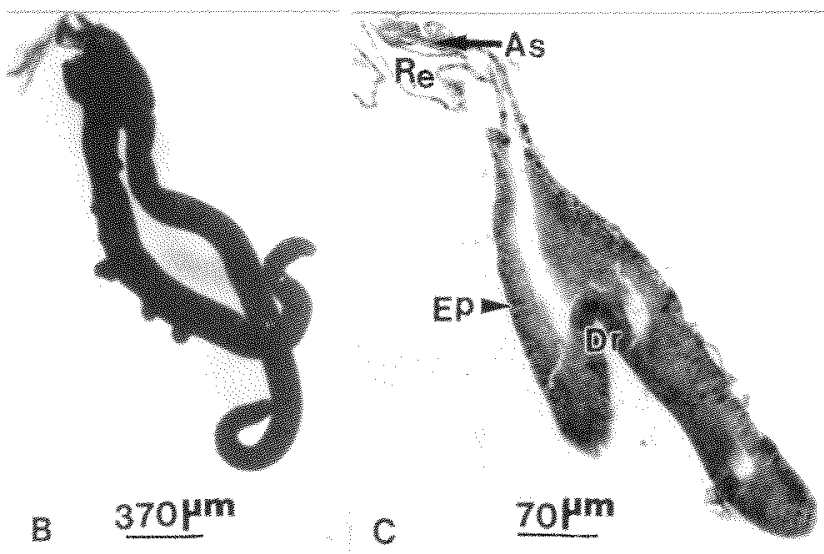

Fig. 2: Panorpa vulgaris Speicheldrüsen. A-B. Totalpräparat. A. Speicheldrüse ohne Divertikel; B. Speicheldrüse mit Divertikel. Boraxcarmin-Färbung; C. Längsschnitt durch die Drüsenschläuche. Azan-Färbung/ Specht. As $=$ Ausführgang, $\mathrm{Ep}=$ Epithelzelle, $\mathrm{Dr}=$ Drüsenschläuche, $\mathrm{Re}=$ Reservoir .

Drüsenzellen zeigen eine merokrine Sekretion. Ein Beginn der Sekretablagerung unter der apikalen Zellmembran ist erkennbar. Das Lumen der Speicheldrüsen der gefütterten Tiere ist im Gegensatz zu den ungefütterten Tieren je nach ihrem Sekretionszustand mehr oder weniger mit homogenem bis granulärem Sekret gefüllt (Fig. 3C). Das Sekret in den Lumina der Drüsenschläuche färbt sich je mit Giemsa rosa, mit Hämalaun-Eosin schwach rosa bis braun, mit Eisenhätoxylin nach Heidenhain schwarz-braun, mit Azan nach Heidenhain intensiv tot bis blau (ROMEIS 1989). Die durchgeführten Färbungen der Semidünnschnitte mit Toluidinblau lassen einzelne Granula des Sekrets deutlich hervortreten.

.Zwischen den Epithelzellen des Reservoirs und des Ausführganges bestehen hinsichtlich ihres zellulären Aufbaus und ihres Färbungsverhaltens keine große Unterschiede (Fig. 4). Beide sind mit einem flachen Epithel versehen, dessen ca. $6 \mu \mathrm{m}$ großen Kerne entsprechend abgeflacht sind. Diese Kerne färben sich mit Azan stark rot; die Zellgrenzen sind nicht deutlich erkennbar. Außen liegt dem Epithel eine dünne Basalmembran an, innen ist es mit einer zarten Intima ausgekleidet. Weder das Reservoir noch der Ausführgang stehen mit Muskeln oder Nerven in direkter Verbindung. 


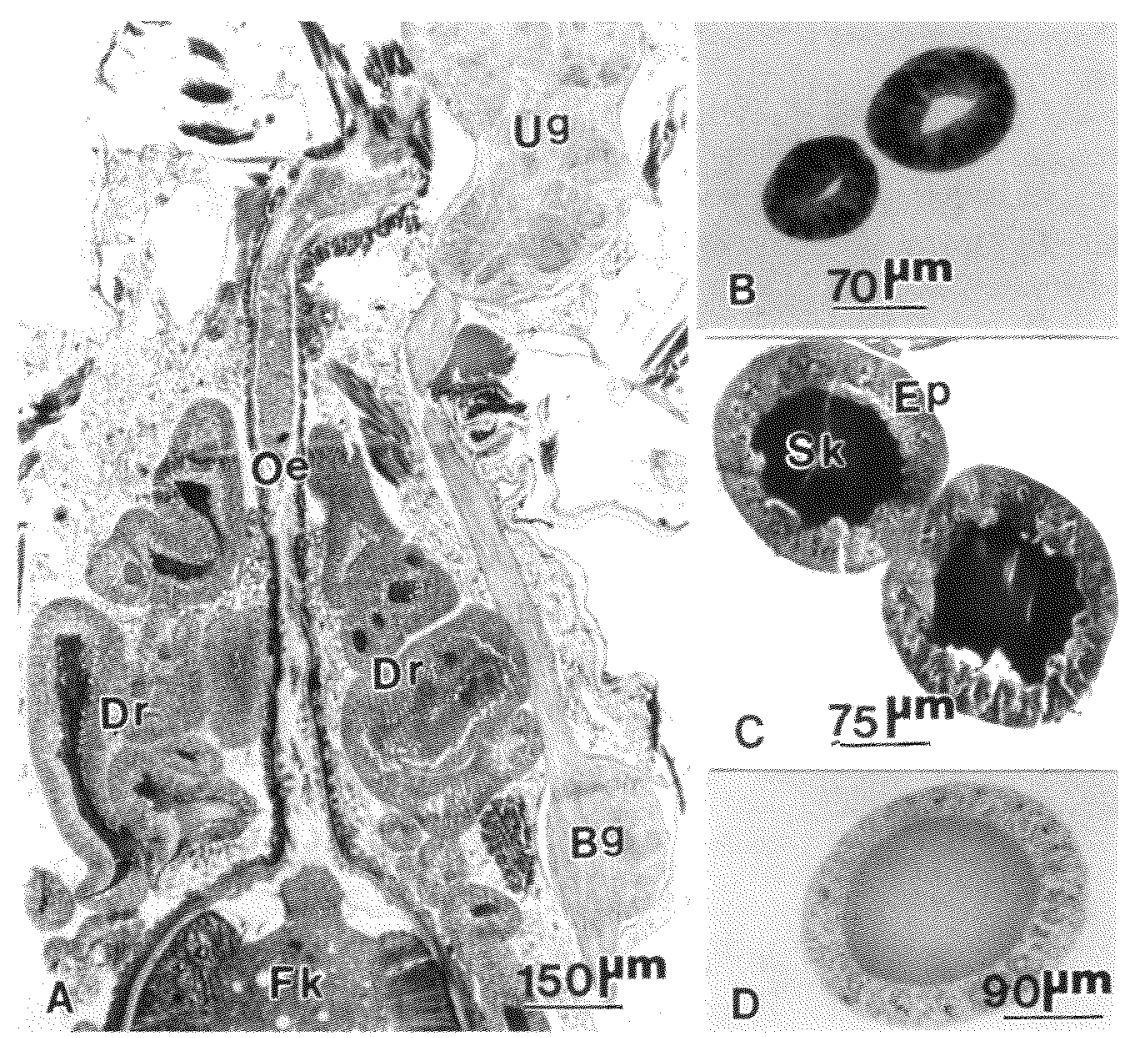

Fig. 3: Panorpa vulgaris Speicheldrüsen. A. Längsschnitt durch die Drüsenschläuche im Oesophagialbereich. Azan-Färbung; B-D. Querschnitt durch die Drüsenschläuche; B. Drüsenschläuche ohne Sekret (ungefüttert). Azan-Färbung; C. Drüsenschläuche mit Sekret (gefüttert). Azan-Färbung; D. Semidünnschnitt. Toluidinblau-Färbung. $\mathrm{Bg}=$ Bauchganglion, $\mathrm{Dr}=$ Drüsenschläuche, $\mathrm{Ep}=$ Epithelzelle, $\mathrm{Oe}=$ Oesophagus, $\mathrm{Sk}=\mathrm{Se}-$ kret, $\mathrm{Ug}=$ Unterschlundganglion.

Die Speicheldrüsen sind bei ungefütterten und gefütterten Tieren gleich lang. Sie sind auch während einer Hungerphase von 3-18 Tagen (nach dem Schlupf der Imago) weiterhin gut entwickelt, zeigen allerdings keine Sekretproduktion.

Wie im histologischen Schnitt erkennbar, ist bei gefütterten Tieren das Lumen des zum Teil gefalteten Ausführganges so stark mit Sekret gefüllt (Fig. 4B), daß es zu einer enormen Dehnung des Ausführganges kommt. An den Ausführgang schließt sich vor der Mündung eine Speichelpumpe an (Fig. 4A), die in ihrem histologischen Aufbau dem Verschlußapparat der Speicheldrüsen von $P$. communis sehr ähnelt. Das Sekret füllt den Ausführgang bis zur Speichelpumpe aus (Fig. 5B). Die Wand der Speichelpumpe wird von einer dorsalen und einer ventralen Platte gebildet. Die Ventralplatte ist sklerotisiert; die Dorsalplatte mit einer Intima ausgekleidet. Sie wird mit Azan nach Heidenhain und Specht stark blau gefärbt. Im Ruhezustand legt sich die Dorsalplatte eng an die Ventralplatte an, so daß das Sekret im Ausführgang nicht weiter olralwärts geschoben werden kann. An der Dorsalplatte setzen drei Muskelstränge an, die, wie histologische Schnitte zeigen, aus zwei dorsolateralen Kontraktions- und einem median gelegenen DilatatorMuskel bestehen. 
Durch Kontraktion dieser Muskeln wird die Dorsalplatte gehoben, so daß das Sekret, das im Ausführgang unter starken Druck steht, in das Lumen der Speichelpumpe gedrückt wird. Dadurch, daß die Muskulatur oralwärts fortschreitend erschlafft, wird das Sekret aus der Speichelpumpe via Mündung nach außen gedrückt. Danach wird das Lumen der Speichelpumpe wieder geschlosssen.

Das Sekret der Drüsenschläuche von P. vulgaris zeigt keine Reaktion auf die histochemische Nachweismethoden für Kohlenhydrate und saure Mucopolysaccharide mit BEST's Karmin, PAS und PAS-Speichel (SPANNHOFF 1967).

Der Nachweis basischer Proteine anhand histochemischer Methoden erbrachte eindeutig positive Ergebnisse. Mit Quecksilber-Bromphenol (RUTHMANN 1966) färbt sich das Sekret intensiv blau, mit Fastg-Green (Romers 1989) zeigt das Sekret selektiv eine grüne Färbung (Fig. 6).

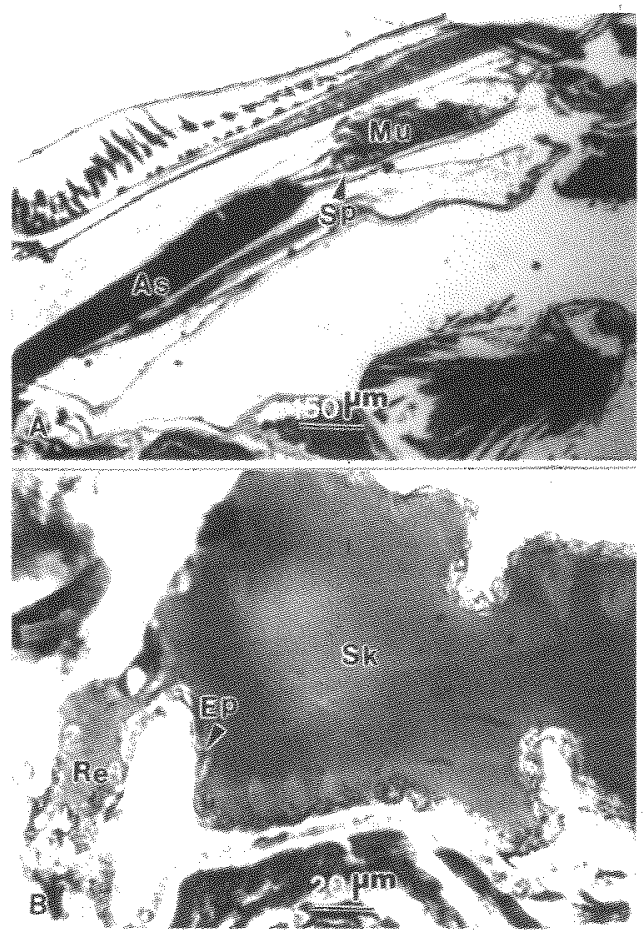

Fig. 4: Panorpa wulgaris Ausführgang der Speischeldrüsen. A. Längschnitt eines sekretgefüllten Ausführganges; B. Sekretgefüllter Ausführgang bei stärkerer Vergrößerung. Azan-Färbung. As = Ausführgang, Ep = Epithelzelle, $\mathrm{Mu}=$ Muskulatur, $\mathrm{Re}=$ Reservoir, $\mathrm{Sk}=$ Sekret, $\mathrm{Sp}=$ Lumen der Speichelpumpe.
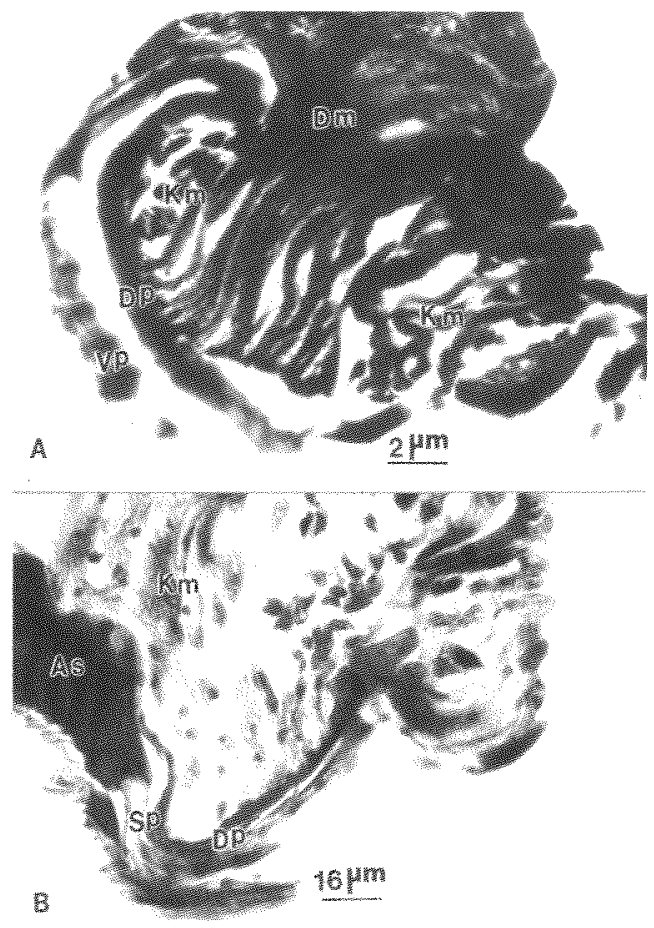

Fig. 5: Panorpa vulgaris Querschnitt durch die Speichelpumpe. A. Ventral- und Dorsalplatte mit Muskulatur. Quecksilber-Bromphenol-Färbung; B. Längsschnitt durch den Ausführgang und die Speichelpumpe. AzanFärbung/Specht. As=Ausführgang mit Sekret, Dm= Dilitatorenmuskel, $\mathrm{D}_{\mathrm{p}}=$ Dorsalplatte, $\mathrm{Km}=$ Kontraktionsmuskel, $\mathrm{Sp}=$ Lumen der Speichelpumpe, $\mathrm{Vp}=$ Ventralplatte. 
Mit Sudanschwarz-B, 1\%iger Osmiumsäure und Scharlach R (= Sudan IV) (SPANNHOFF 1967) konnten am reinem Speicheldrüsensekret von P. vulgaris keine Lipide nachgewiesen werden.

\section{Diskussion}

Die morphologischen und histologischen Untersuchungen zeigen, daß die Drüsenschläuche adulter Individuen der männlichen Skorpionsfliege $P$. vulgaris sowohl bei ungefütterten als auch bei gefütterten Expemplaren gleich stark entwickelt sind. Die Entwicklung und der Füllungsgrad der Drüsen und der Aufbau des Drüsenepithels der Imagines hängt viel mehr von der aufgenommenen Nahrung während der Larvalphase ab. Dieser Befund stimmt mit den Ergebnissen von FLECK u. a. (1994) überein.

Das Lumen der Drüsenschläuche ist bei gefütterten Tieren je nach der Nahrungsverfügbarkeit (Fütterung mit 1 oder 2 Segmenten) unterschiedlich stark mit Sekret gefüllt.

Histologische Schnitte zeigen, daß die Wand des Ausführganges von P.vulgaris sich dehnt, während das Lumen mit Sekret gefüllt wird. GRELL (1963) kommt bei seinen Untersuchungen an $P$. communis zu ähnlichen Befunden.
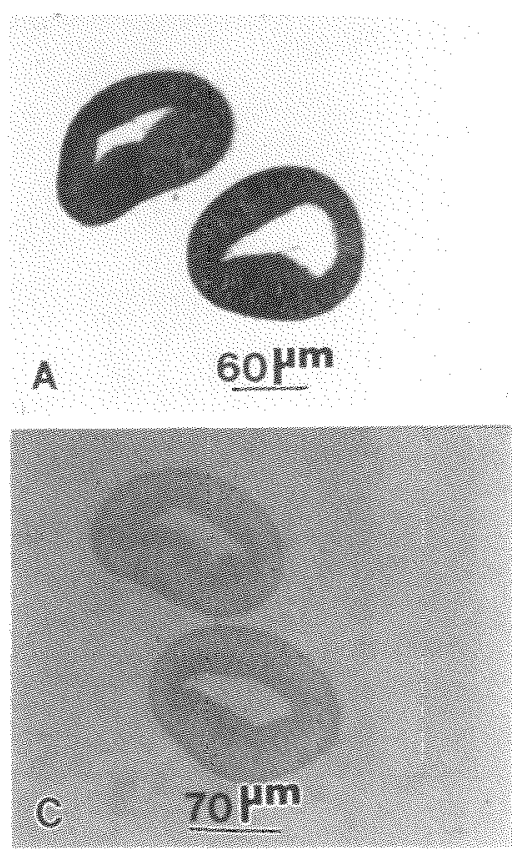

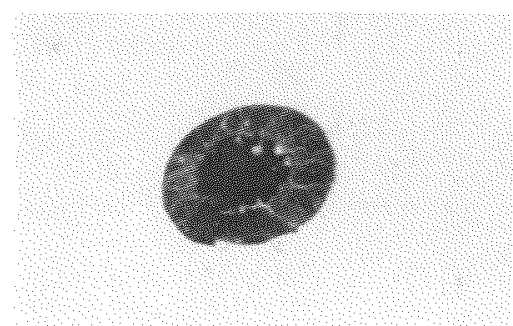

10 inn

B

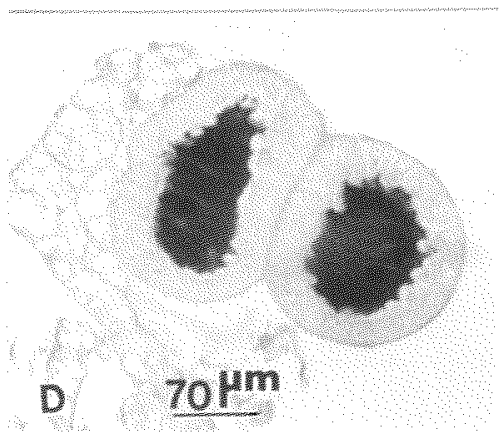

Fig. 6: Panorpa vulgaris Protein-Nachweis an den Speicheldrüsen. A-D. Querschnitt durch die Drüsenschläuche; A. Drüsenschläuche ohne Sekret (nicht gefüttert); B. Drüsenschläuche mit Sektet (gefüttert). Quecksilber-Bromphenol-Färbung; C. Drüsenschläuche ohne Sekret (nicht gefüttert); D. Drüsenschläuche mit Sekret (gefüttert). Fast-Green-Färbung. 
Die Speicheldrüsen von $P$. vulgaris besitzen keine Muskelansätze, die für ein aktives Sezernieren der Sekret-Bonbons sprechen. Sie besitzen aber eine Speichelpumpe, die die Funktion eines Verschlußaparates übernimmt. Diese Speichelpumpe wurde bei $P$. communis als Sekretformer (GRELL 1936) bezeichnet. Ausbau und Funktion der Speichelpumpe von P. vulgaris entspricht der Struktur und den Mechanismus der Speichelpumpe von $P$. communis (GRELL 1936 \& KALTENBACH 1978). Bei gefütterten Tieren von P. vulgaris reicht das mit Azan rot gefärbte Sekret bis zur Speichelpumpe.

Durch Erhöhung des Haemolymphdruckes gelangt das Sekret nach der Öffnung der Speichelpumpe aus den Drüsenschläuche via Reservoir und Ausführgang nach außen.

Das Sekret der Drüsenschläuche von $P$. vulgaris zeigt keine Reaktion auf die Nachweistests für Kohlenhydrate und saure Mucopolysaccharide. Daraus läßt sich schließen, daß im Sekret weder Polysaccharide und noch saure Mucopolysaccharide vorhanden sind. Auch STEINER (1930) fand im Sekret der Speicheldrüsen von P. communis keine Kohlenhydrate.

Anhand spezieller Nachweisverfahren wurde gezeigt, daß das Sekret der Speicheldrüsen von $P$. vulgaris weitgehend aus Proteinen besteht. Mit histochemischen Färbungen konnten basische Proteine im Sekret der Speicheldrüsen von P. vulgaris nachgewiesen werden.

\section{Tab.1: Anzahl der Speicheldrüsenschläuche - prozentuale Verteilung .}

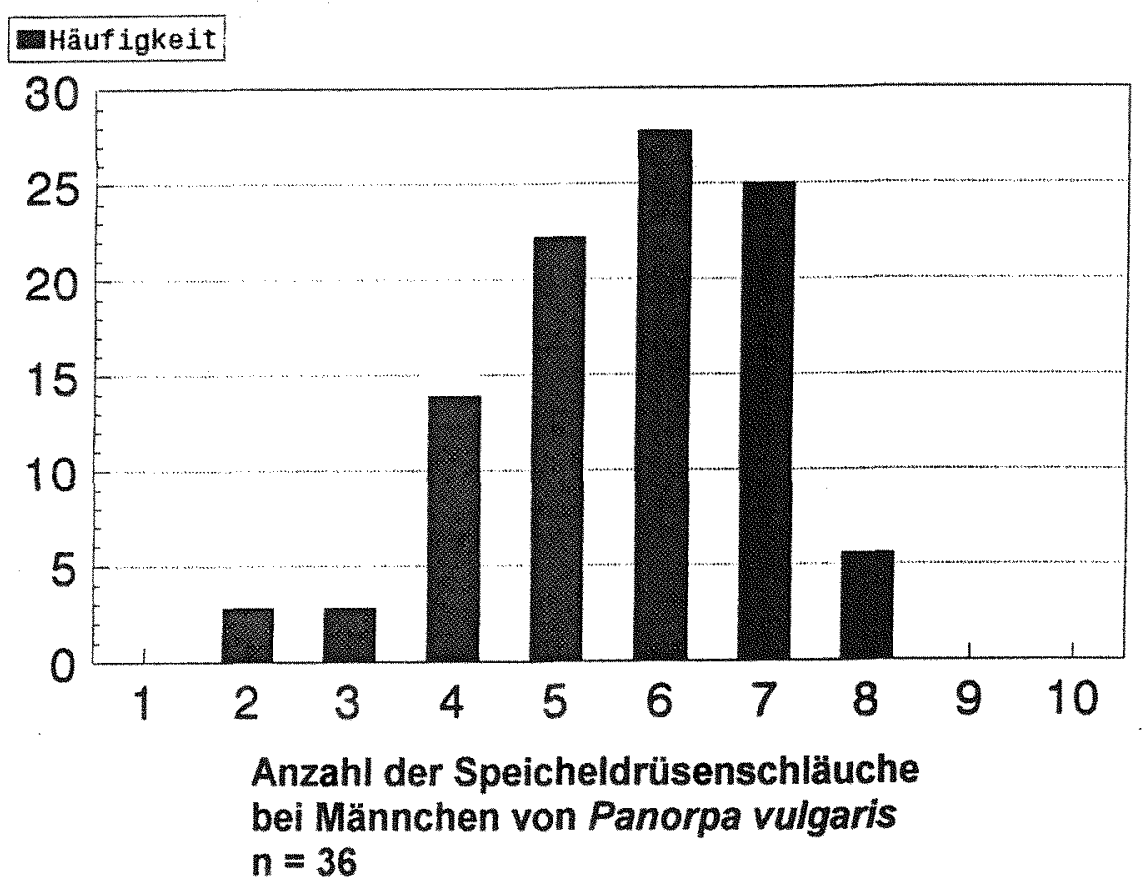


STEINER (1930) fand, daß das Sekret der Speicheldrüse von $P$. communis auch aus proteinhaltigen Substanzen besteht. Auch nach FLECK (1997) enthält das Sekret der Speicheldrüsen von P. vulgaris einen hohen Anteil an Proteinen und essentiellen Aminosäuren.

Die unterschiedlichen des Sekrets auf verschiedene Färbemethoden könnten möglicherweise damit erkläret werden, daß die kolloidale Natur des Sekrets verschiedenen Proteinmustern entspricht.

\section{Literatur}

BYERS, G. W. \& THORnhill, R. 1983: Biology of the Mecoptera. - Ann. Rev. Entomol., California 28: 203-228. FLECK, S.; SINDERN, J. \& SAUER, K. P. 1994: "Nupital feeding" und seine Abhängigkeit von der Nahrungsverfügbarkeit bei der Skorpionsfliege Panorpa vulgaris. - Verhandl. Deutsch. Zool. Ges., Stuttgart 871: 36 .

FLECK, S. 1997: Das Hochzeitsgeschenk der Skorpionsfliege Panorpa vulgaris (Insecta: Mecoptera): Ein betrugssicherer Indikator für die genetische Qualität. - Diss. Arb. Uni. Bonn: $78 \mathrm{~S}$.

GRELL, K. G. 1936: Der Darmtraktus von Panorpa communis L. und seine Anhänge bei Larve und Imago. Zool. Jahrb. Abt. F. Anatomie, Jena 64: 1-85.

KALTENBACH, A. 1978: Mecoptera (Schnabelhafte, Schnabelfliegen). - In: HEIMCKE, STARCK, WERMUTH Handbuch der Zoologie IV. Band: Arthropoda 2. Hefte: Insecta. - Berlin, Verl. Walter de Gruyter.

NAHIF, A. A. \& MADEL, G. 1995: Zur Morphologie und Entwicklung von Panorpa vulgaris (Insecta: Mecoptera, Panorpidae). - Mitt. Dtsch. Ges. Allg. Angew. Ent., Göttingen 10: 609-612.

PLATTNER, H. 1981: In: SCHIMMEL, G. \& W. VOGELL - Methodensammlung der Elektronen-mikroskopie, Wissenschaftliche Verlagsgesellschaft MBH, Stuttgart: 27-28.

Romeis, B. 1989: Mikroskopische Technik. 17. Aufl., München. - Urban \& Schwarzenberg, 577 S.

RUTHMANN, A. 1966: Methoden der Zellforschung. - Francksche Verlagsanstalt, Stuttgart, $301 \mathrm{~S}$.

SAUER, K. P. \& HANSLE, R. 1977: Reproduktive Isolation, ökologische Sonderung und morphologische Differenz der Zwillingsarten $P$. communis L. und P. vulgaris IMHOFF und LABRAM (Insecta, Mecoptera). Z. Zool. Syst. Evolut. Forsch., Berlin 15: 169-207.

Sindern, J; KUlLMANN, H. \& SAUER, K. P. 1995: Evidenz für Partnerwahl durch beide Geschlechter bei der Skorpionsfliege Panorpa wulgaris. - Verh. Dtsch. Zool. Ges., Stuttgart 88: 52.

SPANnhoff, M. 1967: Einführung in die Praxis der Histochemie 2. Aufl., Jena, - G. Fischer Verl.: 172 S.

STEINER, P. 1929: Studien an Panorpa communis L. I. Zur Biologie; II Zur Morphologie und post-embryonalen Entwicklung des Kopfskelettes. - Z. Morphl. Ökol. Tiere, Berlin 17: 1-67.

Weber, H. 1954: Gtundriß der Insektenkunde 3. Aufl., Stuttgart. - G. Fischer Verl.: 428 S.

\section{Anschrift des Verfassers:}

Prof. Dr. AlI AGHA NAHIF

Institut für Evolutionsbiologie und Zooökologie

An der Immenburg 1

D-53121 Bonn

Deutschland 